\title{
Expression and role of HEPIS in breast cancer
}

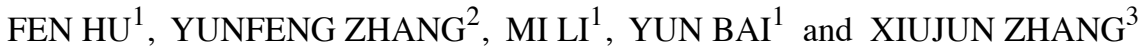 \\ ${ }^{1}$ College of Life Sciences, North China University of Science and Technology, Tangshan, Hebei 063210; \\ ${ }^{2}$ Department of Life Sciences, Tangshan Normal University, Tangshan, Hebei 063000; ${ }^{3}$ College of Psychology, \\ North China University of Science and Technology, Tangshan, Hebei 063210, P.R. China
}

Received September 14, 2018; Accepted September 6, 2019

DOI: $10.3892 / \mathrm{ol} .2019 .10993$

\begin{abstract}
Human embryo lung cellular protein interacting with severe acute respiratory syndrome-coronavirus nonstructural protein-10 (HEPIS) is expressed at varying levels in multiple organs and breast cancer cell lines. However, its expression and function in breast cancer cells has yet to be studied. Therefore, RNA in situ hybridization was used to detect the expression of HEPIS in breast cancer and cancer-adjacent normal breast tissue. HEPIS was expressed at lower levels in breast cancer compared with that in adjacent normal tissue. Subcellular localization of HEPIS was mainly found in the cytoplasm of HeLa cells. Cell Counting Kit-8 and 5-ethynyl-2'-deoxyuridine cell proliferation assays were used to investigate the role of HEPIS in cancer cell proliferation. Ectopic expression of HEPIS in MCF-7 cells was found to significantly inhibit cell proliferation. In contrast, knockdown of HEPIS by RNA interference exhibited the opposite effect. Furthermore, a dual-luciferase reporter assay was performed and HEPIS overexpression specifically inhibited the activity of the NF- $\mathrm{KB}$ reporter gene. Results of the gene chip assay revealed that 2,231 genes were differentially expressed in $H E P I S$-overexpressing cells. Results of the Gene Ontology and Kyoto Encyclopedia of Genes and Genomes enrichment analyses indicated that these genes were enriched in the 'mitogen-activated protein kinase signaling pathway', 'JAK-STAT signaling pathway' and 'focal adhesion'. Reverse transcription-quantitative PCR was used to confirm the expression levels of the differentially expressed genes interleukin 2 receptor subunit $\alpha$ (IL2RA), interferon $\alpha$ and $\beta$ receptor subunit 2 (IFNAR2) and IF $\alpha 8$ (IFNA8). In conclusion, the results of the present study indicated that HEPIS may function as a potential repressor of breast cancer.
\end{abstract}

Correspondence to: Dr Fen Hu, College of Life Sciences, North China University of Science and Technology, 21 Bohai Avenue, Tangshan, Hebei 063210, P.R. China

E-mail: ihufen@126.com

Professor Xiujun Zhang, College of Psychology, North China University of Science and Technology, 21 Bohai Avenue, Tangshan, Hebei 063210, P.R. China

E-mail: xiujunzhang66@126.com

Key words: HEPIS, MCF-7, proliferation, expression

\section{Introduction}

Severe acute respiratory syndrome-coronavirus nonstructural protein-10 (SARS-CoV nsp-10) is the main transcriptase of SARS that cleaves polyproteins orf la $(187-260 \mathrm{kDa})$ and orf lb (345-422 kDa) during infection (1). Human embryo lung cellular protein interacting with SARS-CoV nsp-10 (HEPIS) is a novel gene that was previously isolated from a cDNA library of human embryonic lung tissue and the protein it encodes interacts with SARS-CoV nsp10 (2). The HEPIS gene is expressed as two different isoforms, isoform $\mathrm{A}$ and isoform B, which are 220 and 147 amino acids long, respectively $(2,3)$. Previously, it was demonstrated that HEPIS is differentially expressed in ten different types of cancer, including stomach, liver, and prostate cancer (4). Furthermore, the transcription factors Oct-1, NF- $\kappa \mathrm{B}$ and C-Jun are associated with transcriptional regulation of the HEPIS gene (4). Breast cancer is one of the most common malignancies affecting women worldwide $(5,6)$, with the incidence of 92.8 per 100,000 women in western Europe in 2018 (7). Breast cancer progression is a complex process comprising cell cycle dysregulation (8) and metastasis to distant organs (9). A variety of steroid hormones, such as estrogen and progesterone (10), and the expression of specific genes, such as zinc finger E-box-binding homeobox 1 and matrix metallopeptidases (9), have been attributed to the growth and metastasis of breast cancer cells. A previous study showed that the transcription factor Zinc finger E-box-binding homeobox 1 regulates the expression of the $p 21$ and $C D K 4$ genes to promote breast cancer cell proliferation (11). Bone morphogenetic protein 6 has been found to inhibit breast cancer cell proliferation by targeting microRNA-192 and its direct target RB transcriptional corepressor 1 (12). Clinically, therapeutic interventions for the growth and metastasis of breast cancer remain limited. Our previous study demonstrated that the expression of HEPIS was significantly higher in T-47D compared with ZR-75-30, MDA-MB-231 and MCF-7 cells (4). However, the function of HEPIS in breast cancer cell proliferation has not yet been elucidated, and the elucidation of such a mechanism may provide novel approaches for therapy.

In order to reveal the role of HEPIS in the development of breast cancer, the function of HEPIS in MCF-7 cell proliferation and the regulated genes of HEPIS was investigated in the present study. Our results may provide a basis for establishing a more effective treatment strategy for breast cancer. 


\section{Materials and methods}

Plasmid construction. Full-length HEPIS isoform A and B coding sequence (CDS) was amplified from MCF-7 cDNA using PCR with the following primers: Forward, 5'-TTCAAGCTTATGTCTGCCCATATGTCAGG-3' and reverse, 5'-TAAGGATCCGTCACAGGATTTCTCTAA GTCT-3'. The PCR fragments were run on an agarose gel, photographed, recovered, digested with HindIII (AAGCTT) and BamHI (GGATCC) and cloned into the pEGFP-C3 vector (BD Biosciences). pEGFP-C3-HEPISa plasmid contained the CDS sequence of HEPIS isoform $A$, whereas pEGFP-C3-HEPISb plasmid contained the CDS sequence of HEPIS isoform $\mathrm{B}$.

The partial human HEPIS CDS was amplified from MCF-7 cDNA using PCR with the following primers: Forward, 5'-ATACTCGAGGAAGTGGAGCAGGATGTA-3' and reverse, 5'-ATAGCGGCCGCTCAGTCACAGGATTTCTC-3', and the PCR fragments were cloned into the psiCHECK ${ }^{\mathrm{TM}}-2$ (Promega Corporation) vector using XhoI (CTCGAG) and NotI (GCGGCCGC) restriction sites. This partial human HEPIS CDS sequence contained the targeting site of the small interfering (si)RNAs described below.

The target siRNA sequences for human HEPIS were 5'-GATGCTAACCTCCAAGTTT-3', 5'-GCAAGCAGCAGA AGAGAAA-3' and 5'-AGTTTAGTCCTGCAGAGAT-3'. These oligonucleotides were annealed and ligated into pSilencer 4.1-CMVneo (Ambion; Thermo Fisher Scientific, Inc.) to construct HEPIS-specific siRNA expression plasmids siHEPIS-1, siHEPIS-2, and siHEPIS-3, respectively, according to the manufacturer's protocol. pSilencer 4.1-CMVneo expressing scrambled siRNA (Ambion; Thermo Fisher Scientific, Inc.) was used as si-control.

Cell culture. HeLa and MCF-7 cells (ATCC) were maintained in high glucose DMEM (Gibco; Thermo Fisher Scientific, Inc.) supplemented with $10 \%$ fetal bovine serum (FBS; HyClone; GE Healthcare Life Sciences), penicillin (100 U/ml) and streptomycin $(100 \mathrm{U} / \mathrm{ml})$ and incubated at $37^{\circ} \mathrm{C}$ with $5 \% \mathrm{CO}_{2}$.

Cell transfection. HeLa and MCF-7 cells were transfected with plasmid using Lipofectamine ${ }^{\mathrm{TM}} 2000$ (Thermo Fisher Scientific, Inc.), according to the manufacturer's protocol.

HeLa cells were seeded at a density of $1 \times 10^{4}$ cells/well in 96-well plates, after $24 \mathrm{~h}$ of culture, the cells were transfected with $200 \mathrm{ng}$ /well pEGFP-C3-HEPISa, pEGFP-C3-HEPISb, or pEGFP-C3 for subcellular localization. HeLa cells were seeded at a density of $7 \times 10^{4}$ cells/well in 24 -well plates. After $24 \mathrm{~h}$ of culture, the cells were transfected with $800 \mathrm{ng} /$ well pEGFP-C3, pEGFP-C3-HEPISa or pEGFP-C3-HEPISb plasmids for reverse transcription-quantitative PCR (RT-qPCR).

MCF-7 cells were seeded at a density of $1 \times 10^{4}$ cells/well in 96-well plates. After 24 hof culture, the cells were co-transfected with $100 \mathrm{ng} /$ well of either si-control, siHEPIS-1, siHEPIS-2 or siHEPIS-3 and $100 \mathrm{ng} /$ well psiCHECK ${ }^{\mathrm{TM}}$-2-HEPIS for silencing target site selection; the cells were transfected with $200 \mathrm{ng}$ /well pEGFP-C3-HEPISa, pEGFP-C3-HEPISb, pEGFP-C3, siHEPIS-3 or si-control for Cell Counting Kit-8 (CCK-8) assay; the cells were co-transfected with $80 \mathrm{ng} /$ well of pEGFP-C3-HEPISa, pEGFP-C3-HEPISb,
pEGFP-C3, siHEPIS-3 or si-control, 80 ng/well of pNF- $\kappa$ B-Luc and $40 \mathrm{ng} /$ well pRL-TK for Dual-luciferase reporter (DLR) assay.

MCF-7 cells were seeded at a density of $7 \times 10^{4}$ cells/well in 24-well plates. After $24 \mathrm{~h}$ of culture, the cells transfected with $800 \mathrm{ng} /$ well pEGFP-C3, pEGFP-C3-HEPISa, pEGFP-C3-HEPISb, si-control or siHEPIS-3 plasmids for 5-Ethynyl-2'-deoxyuridine (EdU) cell proliferation assay and RT-qPCR; the cells were transfected with $800 \mathrm{ng} /$ well of either pEGFP-C3-HEPISa, pEGFP-C3-HEPISb or pEGFP-C3 for western blotting. The cells were transfected with $800 \mathrm{ng} /$ well of either pEGFP-C3-HEPISa or pEGFP-C3 for gene chip assay.

Transmembrane domain prediction. The transmembrane domain of HEPIS was predicted by Transmembrane Hidden Markov Model (TMHMM) Server v.2.0 (http://www.cbs.dtu. $\mathrm{dk} /$ services/TMHMM/).

Subcellular localization. HeLa cells were transfected as aforementioned. At $24 \mathrm{~h}$ post-transfection, the cells were stained with DAPI for $30 \mathrm{mins}$ at $37^{\circ} \mathrm{C}$, and visualized under an inverted fluorescent microscope as described previously (13).

Western blotting. MCF-7 cells were transfected with EGFP-C3-HEPISa, pEGFP-C3-HEPISb or pEGFP-C3 as aforementioned. At $24 \mathrm{~h}$ post-transfection, western blotting was performed as described previously (14). Primary antibodies specific for GFP (cat. no. SC-8334; Santa Cruz; 1:1,000 dilution) and $\beta$-actin (cat. no. ab8227; Abcam; 1:1,000 dilution) and a secondary antibody Goat Anti-Rabbit IgG H\&L (HRP) (cat. no. ab6721; Abcam; 1:10,000 dilution) were used.

Silencing target site selection using the psiCHECK ${ }^{T M}-2$ vector. MCF-7 cells were transfected with the psiCHECK ${ }^{\mathrm{TM}}-2$ vector as aforementioned. At $24 \mathrm{~h}$, the cells were lysed and cell lysates were assayed for firefly and Renilla luciferase activity using a Dual-Luciferase Reporter Assay System (Promega Corporation) according to the manufacturer's protocol. Renilla luciferase activity was normalized to firefly luciferase activity. Experiments were performed in triplicate.

CCK-8 assay. MCF-7 cells were transfected as aforementioned. At $24 \mathrm{~h}$, the cells were seeded into 96 -well plates at a density of $2 \times 10^{3}$ cells/well, with six replicates per experiment. The CCK-8 assay (Dojindo Molecular Technologies, Inc.) was performed 1, 2, 3, 4 and 5 days after transfection as described previously (13).

EdU cell proliferation assay. For the EdU assay, MCF-7 cells were transfected as aforementioned. The cells were incubated with $50 \mu \mathrm{M} \mathrm{EdU}$ at $37^{\circ} \mathrm{C}$ for $1 \mathrm{~h} 48 \mathrm{~h}$ after transfection. The cells were fixed with $4 \%$ paraformaldehyde at $37^{\circ} \mathrm{C}$ for $30 \mathrm{~min}$ and stained with $5 \mathrm{mg} / \mathrm{ml}$ Hoechst as described previously (12). Images were captured and EdU-positive cells were calculated as described previously (12).

DLR assay. The $\mathrm{pNF}-\kappa \mathrm{B}-\mathrm{Luc}$ reporter plasmid (PathDetect; Agilent Technologies, Inc.) contains the $\mathrm{NF}-\kappa \mathrm{B}$ response 
element, GGGAATTTCCGGGAATTTCCGGGAACCGGA TTGACCGGCCATGGCGATCGCCCTTAAAGGCCCTTA AAGGCCCTTTTTCCGGGAATTTCC, which was cloned into the 3'UTR of firefly luciferase. pNF- $\kappa B$-Luc was therefore used to measure transcriptional activity of NF- $\mathrm{kB}$. The internal control pRL-TK plasmid contained the Renilla luciferase gene (Promega Corporation). MCF-7 cells transfected with as aforementioned. At $24 \mathrm{~h}$ post-transfection, the DLR assay was performed using Dual-Luciferase Reporter Assay System (Promega Corporation) according to the manufacturer's protocol. Firefly luciferase activity was normalized to Renilla luciferase activity.

RNA in situ hybridization. An RNA microarray containing samples from 39 cases of breast cancer and matching adjacent normal tissue was obtained from Shanxi Chaoying Biotechnology Co., Ltd. (cat. no. BR804a). An antisense probe with a sequence matching part of HEPIS was used: 5'-FITC-TCTGCCCAT ATGTCAGGATTGGAAATAATGGAT-3'. Hybridization was performed as previously described (15). DAPI was used as the counterstain. The stained cells were visualized and images were captured using a laser scanning confocal microscope under consistent excitation wavelength among all groups. The results of the staining were analyzed using ImageJ software version 1.46 (National Institutes of Health).

Gene chip assay. MCF-7 cells were transfected as aforementioned. After $48 \mathrm{~h}$ of transfection, RNA was extracted using TRIzol (cat. no. 15596-018; Invitrogen; Thermo Fisher Scientific, Inc.), and further purified using an RNeasy mini kit (cat. no. 74106) and RNase-Free DNase set (cat. no. 79254; both Qiagen $\mathrm{GmbH}$ ). Total RNA was amplified and labeled using the low input quick amp labeling kit, one-color (cat. no. 5190-2305; Agilent Technologies, Inc.), according to the manufacturer's protocol. Labeled complementary RNA (cRNA) was purified using the RNeasy mini kit (cat. no. 74106; Qiagen $\mathrm{GmbH}$ ) as previously described (16). Each slide (Agilent SurePrint G3 Human Gene Expression Microarray 8x60K) was hybridized with 600 ng Cy3-labeled cRNA using a hybridization kit (cat. no. 5188-5242) and washed in staining dishes with wash buffer kit (cat. no. 5188-5327; all Agilent Technologies, Inc.) $17 \mathrm{~h}$ after hybridization, according to the manufacturer's instructions. Slides were scanned using an Agilent microarray scanner (cat. no. G2565CA; Agilent Technologies, Inc.) with the following default settings: Dye channel, green; scan resolution, $3 \mu \mathrm{m}$; photomultiplier tube, $100 \%$, color depth, 16 bit. Data were extracted using Feature Extraction software v10.7 (Agilent Technologies, Inc.). Raw data were normalized using the quantile algorithm, limma packages in $\mathrm{R}$ version 3.5.3 (http://www.R-project.org/) (17). Two samples were used for gene chip assay.

Gene Ontology (GO) and Kyoto Encyclopedia of Genes and Genomes (KEGG) enrichment analysis. Differentially expressed mRNAs were identified as $\log _{2}$ (fold change) $\mid \geq 2$ and $\mathrm{P}<0.05$. GO enrichment (http://geneontology.org, release number 2019-01-01) and KEGG pathway (http://www.genome. $\mathrm{jp} / \mathrm{kegg}$, release number 91.0, July 2019) analysis of the differentially expressed mRNAs were performed as described previously (18).
Reverse transcription-quantitative PCR (RT-qPCR). MCF-7 and HeLa cells were transfected as aforementioned and $24 \mathrm{~h}$ after transfection, the RNA was extracted using TRIzol ${ }^{\circledR}$ (Invitrogen; Thermo Fisher Scientific, Inc.) and purified using RNeasy mini kit (Qiagen $\mathrm{GmbH})$. Total RNA $(0.5 \mu \mathrm{g})$ from each sample was used for first-strand cDNA synthesis using M-MLV reverse transcription kit (Promega Corporation) according to the manufacturer's protocol. The specific products of human HEPIS were amplified using qPCR with the following primers: HEPIS forward, 5'-GCCAAAGCCCAG TGTTAAAAG-3' and reverse, 5'-GGAGGTTAGCATCAC ATTGTCA-3'; and GAPDH forward, 5'-TGACTTCAACAG CGACACCCA-3' and reverse, 5'-CACCCTGTTGCTGTA GCCAAA-3'. GAPDH was used as an internal control.

MCF-7 cells were transfected with pEGFP-C3-HEPISa or pEGFP-C 3 as aforementioned, and 24 and $48 \mathrm{~h}$ after transfection, RNA extraction, purification and reverse transcription were performed as aforementioned. Specific products of human interleukin 2 receptor subunit $\alpha(I L 2 R A)$, interferon $\alpha$ and $\beta$ receptor subunit 2 (IFNAR2) and IFNA8 genes were amplified using qPCR using the following primers: IL2RA forward, 5'-GGCAGCGGAGACAGAGGAA-3' and reverse, 5'-CCTGGGCGACCATTTAGC-3'; IFNAR2 forward, 5'-TGA TAGCGATACTGAGGC-3' and reverse, 5'-CTGGGATTC TGTAGAGGTG-3'; and IFNA8 forward 5'-GACTCATCT GCTGCTTTG-3' and reverse, 5'-GAATCATTTCCGTGT TGTA-3'. GAPDH was used as an internal control.

Universal PCR Max Mix (Applied Biosystems) was used for qPCR, according to the manufacturer's protocol QPCR was performed using the following thermocycling conditions: $94^{\circ} \mathrm{C}$ for $15 \mathrm{sec}, 60^{\circ} \mathrm{C}$ for $15 \mathrm{sec}$ and $72^{\circ} \mathrm{C}$ for $15 \mathrm{sec}$ for 40 cycles. Quantification was performed using the $2^{-\Delta \Delta \mathrm{Cq}}$ method as described previously (19).

Statistical analysis. Data are presented as the mean \pm standard deviation. SPSS v9.0 software (SPSS, Inc.) was used for statistical analysis. The data of RT-qPCR and EdU cell proliferation assay were analyzed using Student's t-test, the CCK-8 data were analyzed using one-way ANOVA followed by a Dunnett's post hoc test. Optical density or integrated optical density of breast cancer or normal breast tissue was analyzed using Student's t-test. $\mathrm{P}<0.05$ was considered to indicate a statistically significant difference. Data represent three independent experiments.

\section{Results}

Characterization and subcellular localization of HEPIS. HEPIS exists in two different isoforms, A and B. PCR was performed to determine the expression levels of the $\mathrm{A}$ and $\mathrm{B}$ isoforms in MCF-7 cells. The results revealed that both the isoforms were expressed in MCF-7 cells. Isoform A was the most abundant form of HEPIS, based on the intensity of the band (Fig. 1A). Isoforms A and B consist of 220 and 147 amino acids, respectively (Fig. 1B), with predicted molecular masses of 28 and $18 \mathrm{kDa}$, respectively. Protein analyses on TMHMM revealed that the HEPIS protein has no predicted transmembrane domain, suggesting that it may be a soluble protein. To determine its subcellular localization, cDNAs of both the isoforms were fused to GFP in the pEGFP-C3 vector and 
A

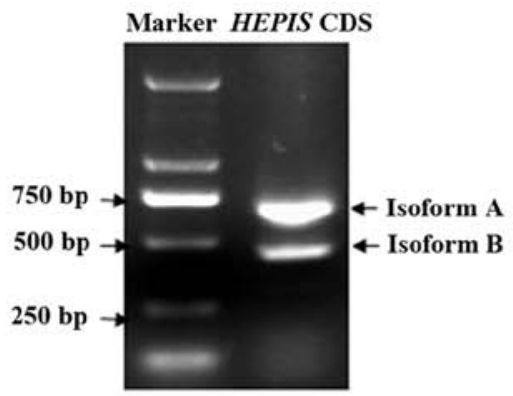

C

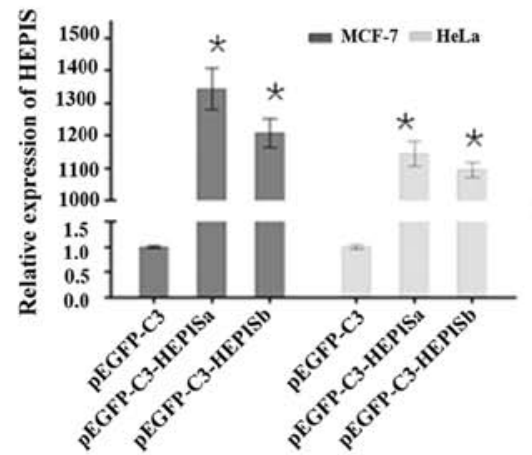

B

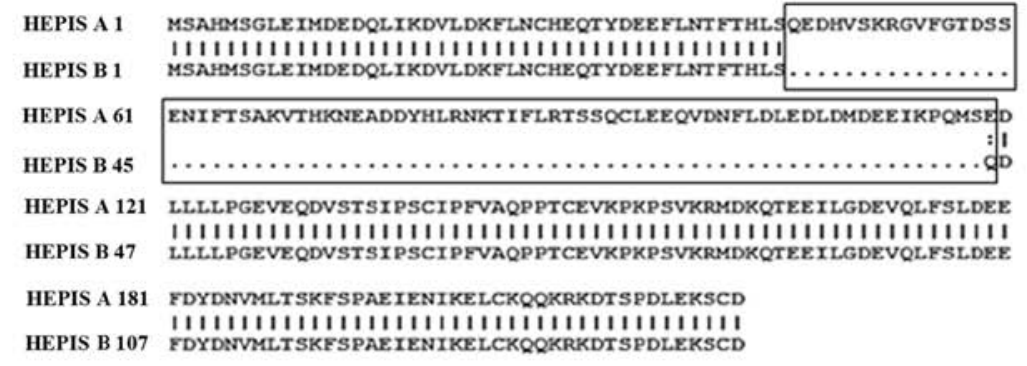

D

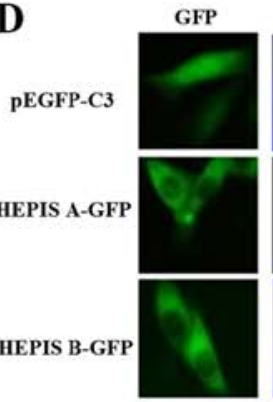

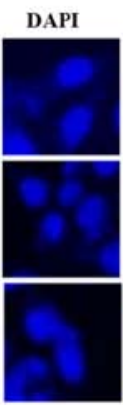

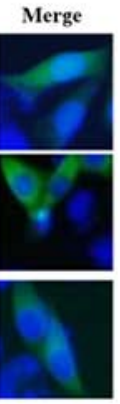

$\mathbf{E}$
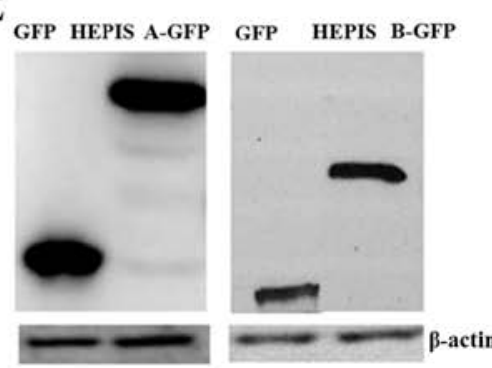

Figure 1. Characterization and subcellular localization of HEPIS. (A) HEPIS mRNA expression levels (isoforms A and B) in MCF-7 breast cancer cells detected using PCR and gel electrophoresis. (B) Protein sequence alignment of HEPIS isoforms A and B. The box indicates different amino acid sequences. Amino acids are presented in a single letter code. (C) HeLa and MCF-7 cells were transfected with pEGFP-C3, pEGFP-C3-HEPISa, or pEGFP-C3-HEPISb. The HEPIS mRNA levels were detected using quantitative PCR $24 \mathrm{~h}$ after transfection. ${ }^{*} \mathrm{P}<0.05$ vs. pEGFP-C3. (D) HeLa cells were transfected with pEGFP-C3, pEGFP-C3-HEPISa, or pEGFP-C3-HEPISb, and nuclei were stained with DAPI was used to stain nuclei. GFP and DAPI staining were visualized using fluorescence microscopy. Magnification, x10. (E) MCF-7 cells were transfected with pEGFP-C3, pEGFP-C3-HEPISa or pEGFP-C3-HEPISb. Western blot analysis with anti-GFP antibodies was performed to determine the expression of HEPIS-GFP in MCF-7 cells. HEPIS, human embryo lung cellular protein interacting with severe acute respiratory syndrome-coronavirus nonstructural protein-10; CDS, coding sequence; GFP, green fluorescence protein.

transfected into HeLa cells. Overexpression of HEPISa and HEPISb was confirmed using qPCR (Fig. 1C). HEPISa-GFP and HEPISb-GFP fusion proteins were primarily expressed in the cytoplasm of HeLa cells (Fig. 1D). Results of the western blot analysis revealed that pEGFP-C3-HEPISa expressed a 55-kDa HEPISa-GFP fusion protein, and pEGFP-C3-HEPISb plasmids expressed a $45-\mathrm{kDa}$ HEPISb-GFP fusion protein in MCF-7 cells (Fig. 1E).

HEPIS expression in breast cancer tissue. In our previous study, it was reported that HEPIS was differentially expressed in T-47D, ZR-75-30, MDA-MB-231 and MCF-7 breast cancer cell lines (4). In the present study, RNA in situ hybridization was performed using a tissue microarray containing breast cancer tissue and adjacent normal tissue from 39 cases to study the expression of HEPIS. HEPIS-positive staining was primarily found in the cytoplasm of case 2 (Fig. 2). The integrated optical density of HEPIS was significantly lower $(\mathrm{P}<0.05)$ in malignant breast cancer tissue compared with the adjacent normal breast tissue (Table I).

HEPIS overexpression reduces proliferation of MCF-7 cells. A previous study reported that HEPIS reduces the proliferation of HeLa cell (2). To assess the effect of HEPIS on the proliferation of breast cancer cells, MCF-7 cells were transfected with the HEPIS-expressing plasmids pEGFP-C3-HEPISa or pEGFP-C3-HEPISb, and a CCK-8 assay was performed. As shown in Fig. 3A, the results of the CCK- 8 assay indicated

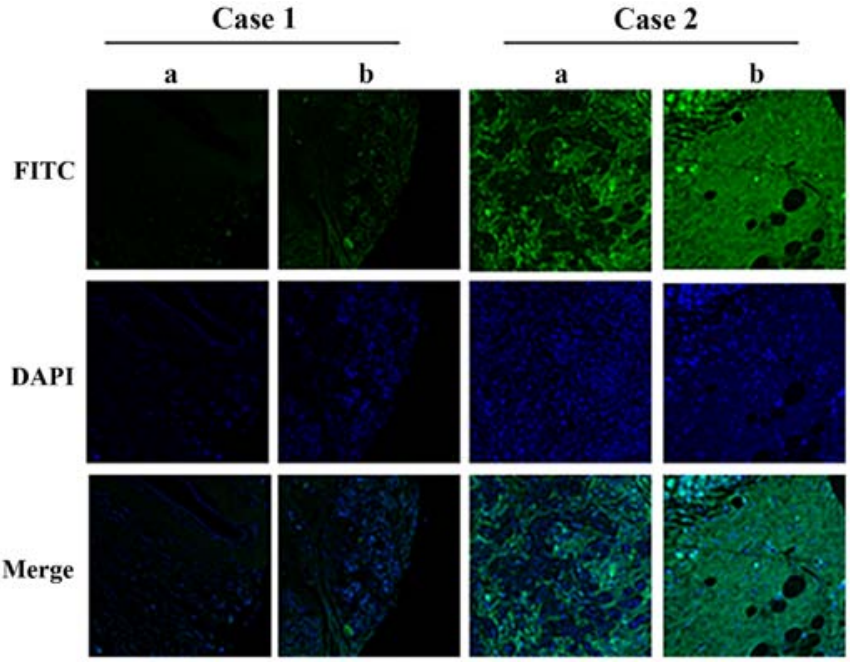

Figure 2. Representative images of RNA in situ hybridization of HEPIS in breast cancer and cancer-adjacent normal breast tissue from two cases. Magnification, $\mathrm{x} 400$. a, invasive ductal carcinoma; b, adjacent normal breast tissue. DAPI was used as the counterstain. HEPIS, human embryo lung cellular protein interacting with severe acute respiratory syndrome-coronavirus nonstructural protein-10.

that overexpression of both isoforms of HEPIS in MCF-7 cells significantly decreased the cell proliferation compared with MCF-7 cells transfected with empty vector controls. To test the effect of knockdown of endogenous HEPIS expression 
Table I. Expression of HEPIS in breast cancer and normal breast tissue specimens.

\begin{tabular}{lccc}
\hline Specimen & Number & Optical density & Integrated optical density \\
\hline Normal breast tissue & 39 & $0.038 \pm 0.001^{\mathrm{a}}$ & $187 \pm 13.51^{\mathrm{a}}$ \\
Breast cancer & 39 & $0.020 \pm 0.001$ & $109 \pm 10.72$ \\
\hline
\end{tabular}

${ }^{\text {aP }}<0.05$, Student's t-test. Data are presented as the mean \pm standard deviation. HEPIS, human embryo lung cellular protein interacting with severe acute respiratory syndrome-coronavirus nonstructural protein-10.

A

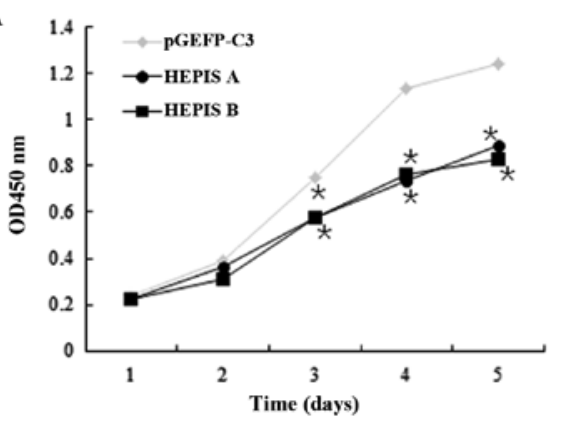

C
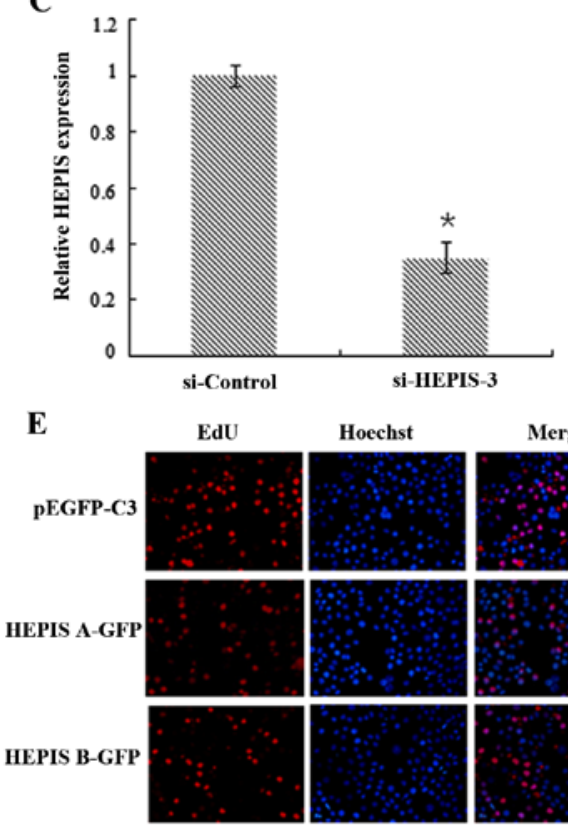

F

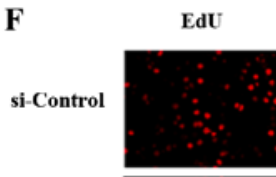

si-HEPIS-3

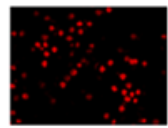

Hoechst
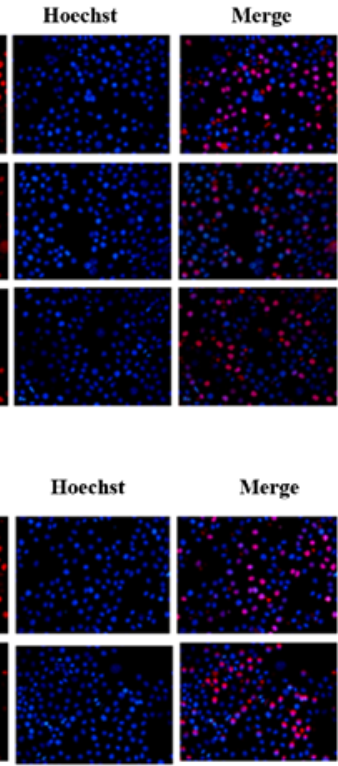

B

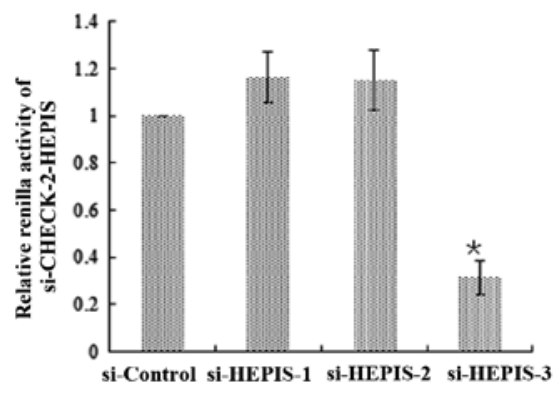

D
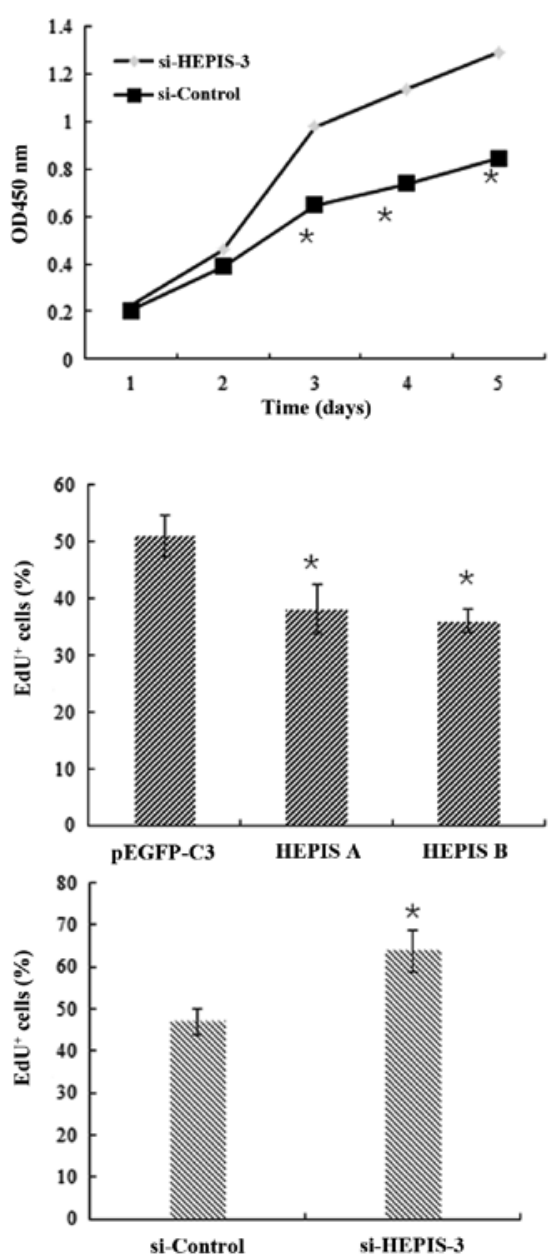

Figure 3. HEPIS inhibits the proliferation of MCF-7 breast cancer cells. (A) CCK-8 assay of MCF-7 cells transfected with pEGFP-C3, pEGFP-C3-HEPISa, or pEGFP-C3-HEPISb. "P<0.05 vs. pEGFP-C3. (B) MCF-7 cells were co-transfected with siHEPIS-1, siHEPIS-2, siHEPIS-3 or si-control, with psiCHECK-2-HEPIS The dual-luciferase reporter assay was performed, and the Renilla luciferase data are normalized to the expression of firefly luciferase. ${ }^{*} \mathrm{P}<0.05$ vs. si-control. (C) MCF-7 cells were transfected with either si-control or siHEPIS-3. The HEPIS mRNA levels were detected using quantitative PCR, $24 \mathrm{~h}$ after transfection. ${ }^{*} \mathrm{P}<0.05$. (D) CCK-8 assay of MCF-7 cells transfected with either si-control or siHEPIS-3. ${ }^{*} \mathrm{P}<0.05$. (E) MCF-7 cells were transiently transfected with pEGFP-C3, pEGFP-C3-HEPISa or pEGFP-C3-HEPISb. Cell proliferation was measured using immunofluorescence analysis of EdU incorporation. "P<0.05 vs. pEGFP-C3. (F) MCF-7 cells were transiently transfected with si-control or siHEPIS-3. Cell proliferation was measured by immunofluorescence analysis of EdU incorporation. ${ }^{*} \mathrm{P}<0.05$ vs. si-control. CCK-8, Cell Counting Kit-8; HEPIS, human embryo lung cellular protein interacting with severe acute respiratory syndrome-coronavirus nonstructural protein-10; EdU, 5-Ethynyl-2'-deoxyuridine; GFP, green fluorescence protein; si, small interfering. 

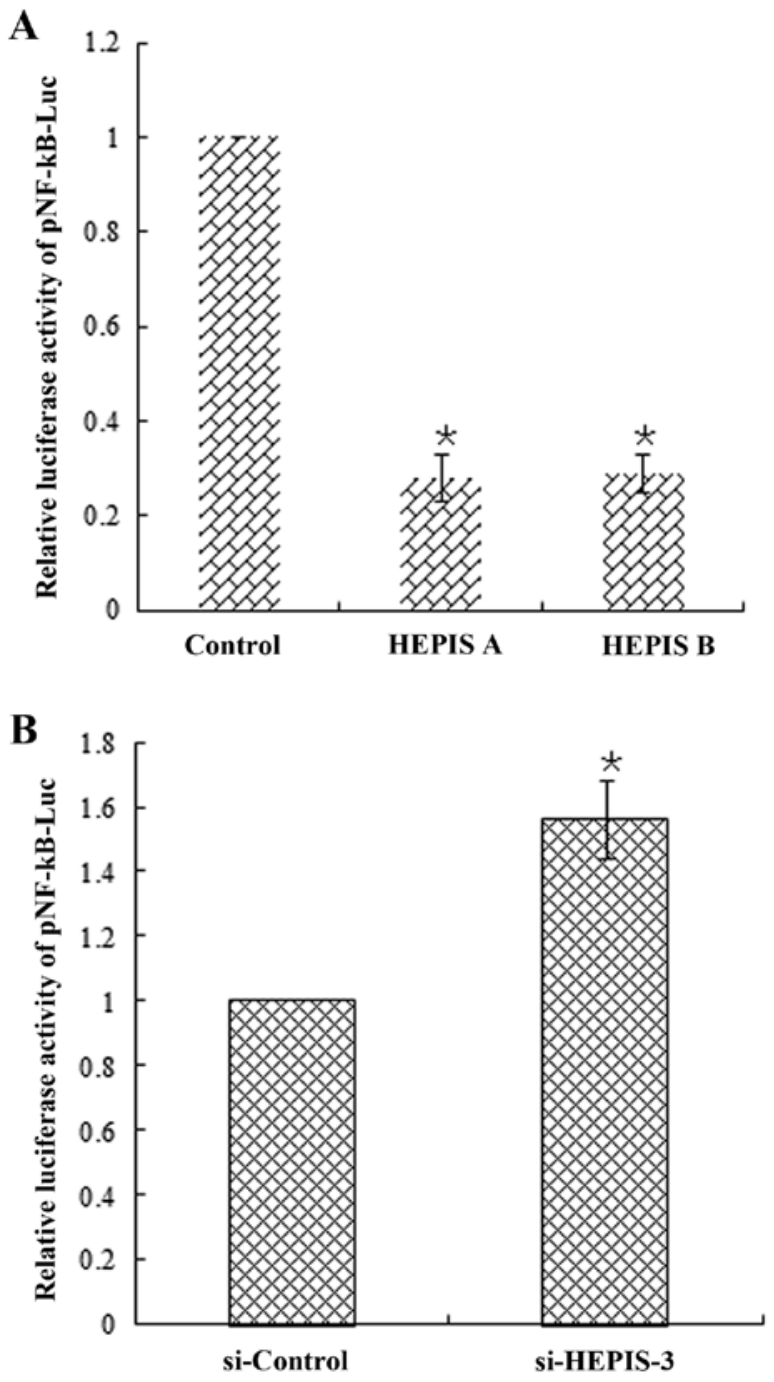

Figure 4. HEPIS inhibits NF- $\mathrm{kB}$ activation in MCF-7 cells. MCF-7 cells were transfected with either (A) pEGFP-C3, pEGFP-C3-HEPISa, or pEGFP-C3-HEPISb and (B) si-control or siHEPIS-3, along with pNF- $\mathrm{kB}-\mathrm{luc}$ $(80 \mathrm{ng})$. The dual-luciferase reporter assay was performed using lysates from transfected cells. "P<0.05 vs. respective control. HEPIS, human embryo lung cellular protein interacting with severe acute respiratory syndrome-coronavirus nonstructural protein-10; si, small interfering; luc, luciferase.

on MCF-7 cell proliferation, three different siRNA plasmids which targeted human HEPIS were constructed (siHEPIS-1, siHEPIS-2 and siHEPIS-3), and the ability of these three plasmids to inhibit HEPIS gene expression using a DLR assay with the psiCHECK ${ }^{\mathrm{TM}}-2$ vector was performed. The results revealed that HEPIS expression was lowest in cells transfected with siHEPIS-3 (Fig. 3B). Results of the qPCR analysis showed that overexpression of siHEPIS-3 significantly reduced the expression of HEPIS at the transcriptional level (Fig. 3C). As shown in Fig. 3D, the knockdown of HEPIS by siHEPIS-3 significantly increased the number of MCF-7 cells compared with the control, confirming that HEPIS has a growth-inhibiting effect on MCF-7 cells. Furthermore, the results of the EdU proliferation assay revealed that overexpression of both isoforms of HEPIS decreased the number of cells in the $\mathrm{S}$ phase from 51.2 to $38.1 \%$ (HEPISa) or 36.3\% (HEPISb) (Fig. 3E). In contrast, HEPIS knockdown resulted in a marked increase in the number of cells in the $\mathrm{S}$ phase, showing an increase
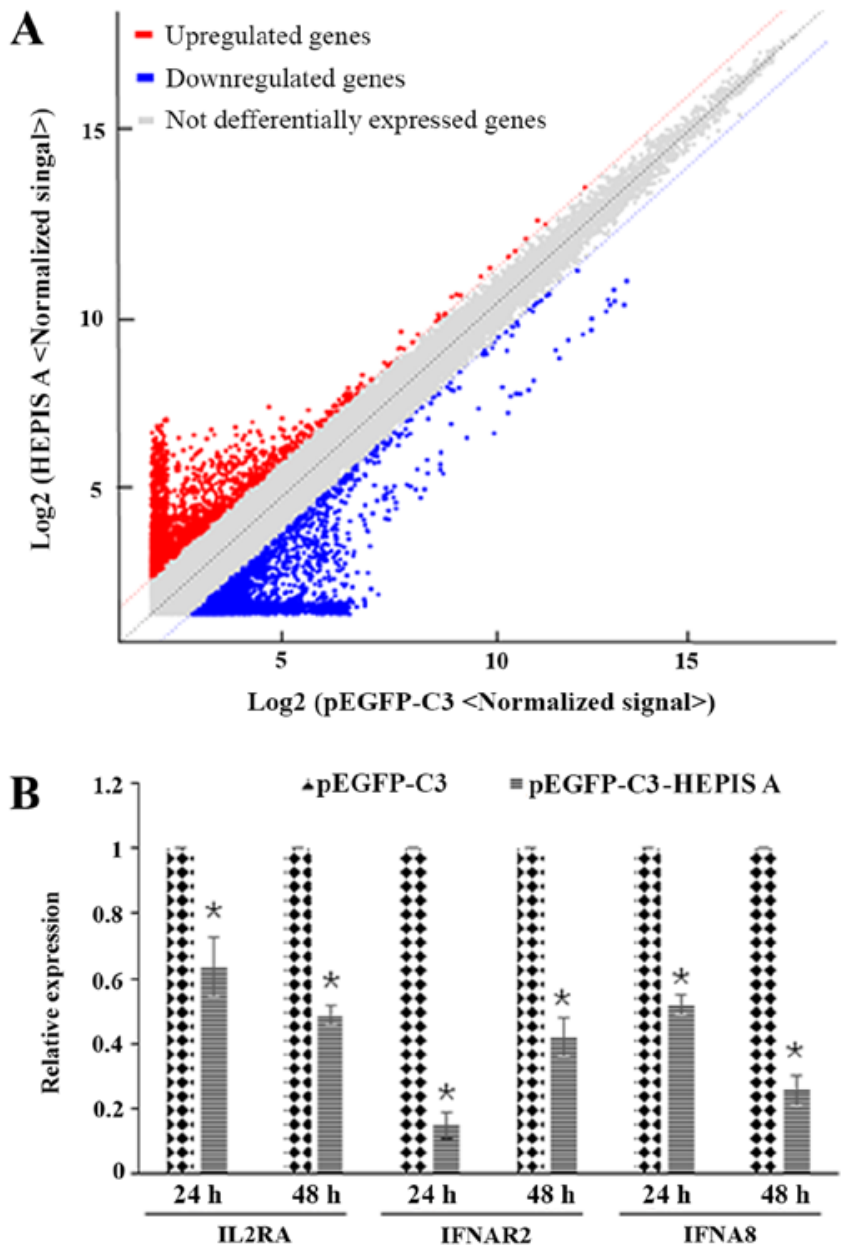

Figure 5. Gene profiles and detection of differentially expressed mRNA. (A) Scatter plot of the expression levels of all the detected mRNAs in HEPIS-overexpressing MCF-7 cells and pEGFP-C3-expressing MCF-7 cells. (B) MCF-7 cells were transfected with either pEGFP-C3, or pEGFP-C3-HEPISa. The IL2RA, IFNAR2 and IFNA 8 mRNA levels were detected using quantitative PCR. ${ }^{*} \mathrm{P}<0.05$ vs. pEGFP-C3. HEPIS, human embryo lung cellular protein interacting with severe acute respiratory syndrome-coronavirus nonstructural protein-10. GFP, green fluorescent protein.

from 47.1 to $63.8 \%$ (Fig. 3F). Based on the above results, it was confirmed that HEPIS inhibits MCF-7 cell proliferation.

HEPIS overexpression inhibits the transcriptional activity of $N F-\kappa B$. The DLR assay was performed to further identify the potential cellular pathways regulated by HEPIS, which revealed that the two isoforms of HEPIS overexpression significantly inhibited the NF-kB reporter gene in MCF-7 cells compared with the control group (Fig. 4A). By contrast, HEPIS knockdown resulted in a significant increase in NF- $\mathrm{KB}$ reporter gene activity (Fig. 4B).

Gene profiles and detection of differentially expressed $m R N A s$. To understand the molecular changes following HEPIS overexpression, gene chip analysis was performed to identify the mRNAs that were differentially expressed between the HEPIS-overexpressing MCF-7 cells and control cells. Results of the gene chip assay showed that 2,231 genes were differentially expressed in HEPIS-overexpressing cells, 
Table II. Top 10 pathways from GO enrichment analysis.

\begin{tabular}{|c|c|c|c|c|c|c|}
\hline GO ID & Description & Type & P-value & $\begin{array}{l}\text { Gene } \\
\text { count }\end{array}$ & Genes & $\begin{array}{l}\text { Enrichment } \\
\text { factor }\end{array}$ \\
\hline GO:0017121 & Phospholipid scrambling & $\mathrm{BP}$ & 0.0014 & 4 & ANO7, P2RX7, PLSCR2, ANO3 & 7 \\
\hline GO:0002664 & $\begin{array}{l}\text { Regulation of } \mathrm{T} \text { cell tolerance } \\
\text { induction }\end{array}$ & BP & 0.0014 & 4 & HLA-B, CD86, CBLB, IL2RA & 7 \\
\hline GO:0042659 & Regulation of cell fate specification & $\mathrm{BP}$ & 0.0018 & 4 & FGF2, POU5F1, GFI1, DKK1 & 6.5 \\
\hline GO:0070307 & Lens fiber cell development & BP & 0.0018 & 4 & FGFR3, FGFR2, WNT7A, WNT7B & 6.5 \\
\hline GO:0002517 & $\mathrm{T}$ cell tolerance induction & $\mathrm{BP}$ & 0.0018 & 4 & HLA-B, CD86, CBLB, IL2RA & 6.5 \\
\hline GO:0004675 & $\begin{array}{l}\text { Transmembrane receptor protein } \\
\text { serine/threonine kinase activity }\end{array}$ & MF & 0.0031 & 4 & $\begin{array}{l}\text { ACVR1, ACVR1B, AMHR2, } \\
\text { TGFBR3 }\end{array}$ & 5.69 \\
\hline GO:0010888 & Negative regulation of lipid storage & $\mathrm{BP}$ & 0.0031 & 4 & TNF, PPARA, NR1H2, ABCA1 & 5.69 \\
\hline GO:0043552 & $\begin{array}{l}\text { Positive regulation of } \\
\text { phosphatidylinositol 3-kinase activity }\end{array}$ & $\mathrm{BP}$ & 0.0002 & 7 & $\begin{array}{l}\text { TGFB1, CCL19, FGF2, PDGFB, } \\
\text { FGFR3, FLT1, CCL21 }\end{array}$ & 5.69 \\
\hline GO:0010560 & $\begin{array}{l}\text { Positive regulation of glycoprotein } \\
\text { biosynthetic process }\end{array}$ & $\mathrm{BP}$ & 0.0031 & 4 & CCL19, PXYLP1, CCL21, SLC51B & 5.69 \\
\hline GO:0043551 & $\begin{array}{l}\text { Regulation of phosphatidylinositol } \\
\text { 3-kinase activity }\end{array}$ & $\mathrm{BP}$ & 0.0001 & 9 & $\begin{array}{l}\text { CCL19, PDGFB, FGFR3, PIK3R5, } \\
\text { CCL21, KIAA0226, TGFB1, FGF2, } \\
\text { FLT1 }\end{array}$ & 5.39 \\
\hline
\end{tabular}

GO, Gene Ontology; BP, biological process; MF, molecular function.

including 1,095 genes that were upregulated and 1,136 that were downregulated (Fig. 5A). The differentially expressed genes were analyzed using GO enrichment and KEGG pathway analysis. Top 10 significant enrichment pathways from GO enrichment analysis included 'transmembrane receptor protein serine/threonine kinase activity' and 'regulation of T cell tolerance induction', among others (Table II). Top 15 significant enrichment pathways from KEGG pathways are listed in Table III and included 'mitogen-activated protein kinase (MAPK) signaling pathway', 'Janus kinase-signal transducer and activator of transcription (JAK-STAT) signaling pathway', 'focal adhesion' and others. The IL2RA, IFNAR2, and IFNA8 genes were selected for qPCR analysis, as they were enriched in the JAK-STAT signaling pathway, from the KEGG enrichment analysis. qPCR results confirmed that the mRNA levels of these three genes were downregulated in HEPIS-overexpressing MCF-7 cells compared with that in the pEGFP-C 3 group at 24 and $48 \mathrm{~h}$ post-transfection (Fig. 5B).

\section{Discussion}

Previously, the HEPIS isoform B has been identified as 147 amino acid long protein with several casein kinase II phosphorylation sites (2). In the present study, HEPIS isoforms A and B were both found to be expressed in MCF-7 cells. Of these, isoform A was the more abundantly expressed isoform. Similar to the present study, a previous study also showed that overexpression of HEPIS isoform B reduced the proliferation of HeLa cells (2). In the present study, it was demonstrated that overexpression of both HEPIS isoforms significantly inhibited cell cycle progression in MCF-7 breast cancer cells by inhibiting G1-S transition, and RNA interference targeting
HEPIS exhibited resulted in increased cell cycle progression, confirming the potential role of HEPIS in the regulation of cell proliferation. Our group previously reported that HEPIS expression was significantly increased in four breast cancer cell lines: T-47D, ZR-75-30, MDA-MB-231, and MCF-7 (4). In addition, the HEPIS expression levels were 8-fold higher in the T-47D cell line compared with that in the MCF-7 cell line (4). Cell proliferation is a complex process that is regulated by multiple genes, such as c-Myc and cyclin D1 (8). The difference in expression of the HEPIS gene does not completely determine the growth rate of MCF-7 and T-47D cells. HEPIS in esophageal squamous cell carcinoma exhibits upregulated expression compared with adjacent normal tissue, whereas in rectal adenocarcinoma, the reverse has been observed; therefore HEPIS may possess varying functions in different types of cancer, due to each cancer having its own complex mechanism (4). In the present study HEPIS expression was down-regulated in breast cancer tissue compared with the adjacent normal tissue.

$\mathrm{NF}-\kappa \mathrm{B}$ belongs is a member the Rel/NF- $\mathrm{KB}$ family, and it is a hetero- or homo-dimeric transcription factor $(20,21)$. NF- $\kappa \mathrm{B}$ serves a vital role in cell proliferation and apoptosis $(20,22)$. The results of the present study demonstrated that HEPIS inhibits the activity of the NF- $\mathrm{KB}$ reporter gene. HEPIS may serve an important role by regulating NF- $\kappa \mathrm{B}$ signal pathway.

The JAK-STAT signaling pathway transmits information from extracellular signals, including a wide array of cytokines (IFN- $\alpha$, IFN- $\gamma$, etc.) and growth factors (epidermal growth factor, platelet-derived growth factor, etc.) (23). This pathway plays a role in proliferation, migration and apoptosis $(24,25)$, and disruption or dysregulation of this pathway can result in prostate cancer (26) or breast cancer (27). MAPKs are a highly conserved family of serine/threonine protein kinases, and are 
Table III. Top 15 pathways from Kyoto Encyclopedia of Genes and Genomes enrichment analysis.

\begin{tabular}{|c|c|c|c|c|c|}
\hline Pathway ID & Description & P-value & $\begin{array}{l}\text { Gene } \\
\text { count }\end{array}$ & Genes & $\begin{array}{l}\text { Enrichment } \\
\text { factor }\end{array}$ \\
\hline hsa00780 & 'Biotin metabolism' & 0.0152 & 1 & BTD & 7.83 \\
\hline hsa04320 & 'Dorso-ventral axis formation' & 0.0031 & 5 & PIWIL3, MAPK3, ETV7, ETS1, NOTCH1) & 4.19 \\
\hline hsa04976 & 'Bile secretion' & 0.0007 & 10 & $\begin{array}{l}\text { ABCC2, SLCO1A2, ABCG5, SLC9A1, } \\
\text { SLC10A1, EPHX1, etc. }\end{array}$ & 3.31 \\
\hline hsa05330 & 'Allograft rejection’ & 0.0133 & 5 & $\begin{array}{l}\text { HLA-DQB1, CD86, HLA-DQA1, HLA-B, } \\
\text { TNF }\end{array}$ & 3.09 \\
\hline hsa05332 & 'Graft-vs.-host disease' & 0.0189 & 5 & $\begin{array}{l}\text { HLA-DQB1, CD86, HLA-DQA1, HLA-B, } \\
\text { TNF }\end{array}$ & 2.86 \\
\hline hsa04940 & 'Type I diabetes mellitus' & 0.0234 & 5 & $\begin{array}{l}\text { HLA-DQB1, CD86, HLA-DQA1, HLA-B, } \\
\text { TNF }\end{array}$ & 2.73 \\
\hline hsa00770 & 'Pantothenate and CoA biosynthesis' & 0.0867 & 2 & DPYS, PPCDC & 2.61 \\
\hline hsa04660 & 'T cell receptor signaling pathway' & 0.0052 & 11 & $\begin{array}{l}\text { PAK7, PIK3R5, CBLC, PTPRC, MAPK3, } \\
\text { etc. }\end{array}$ & 2.46 \\
\hline hsa04930 & ‘Type II diabetes mellitus’ & 0.0379 & 5 & $\begin{array}{l}\text { CACNA1B, CACNA1G, PIK3R5, } \\
\text { MAPK3, TNF }\end{array}$ & 2.45 \\
\hline hsa05211 & 'Renal cell carcinoma' & 0.0192 & 7 & $\begin{array}{l}\text { PAK7, PIK3R5, MAPK3, ETS1, TGFB1, } \\
\text { FLCN, etc. }\end{array}$ & 2.45 \\
\hline hsa04630 & 'JAK-STAT signaling pathway' & 0.0211 & 13 & $\begin{array}{l}\text { IFNL2, IL2RA, LEP, IFNAR2, IFNA8, } \\
\text { etc. }\end{array}$ & 2.4 \\
\hline hsa04672 & $\begin{array}{l}\text { 'Intestinal immune network for } \\
\text { IgA production' }\end{array}$ & 0.4137 & 5 & $\begin{array}{l}\text { HLA-DQB1, CXCL12, TGFB1, CD86, } \\
\text { HLA-DQA1 }\end{array}$ & 1.93 \\
\hline hsa04510 & 'Focal adhesion' & 0.0359 & 15 & $\begin{array}{l}\text { PXN, PAK7, FLT1, ZYX, ACTN2, } \\
\text { COMP, etc. }\end{array}$ & 1.73 \\
\hline hsa04010 & 'MAPK signaling pathway' & 0.0364 & 18 & $\begin{array}{l}\text { NGF, FGF3, NR4A1, FGFR2, FGF2, } \\
\text { FGFR3, etc. }\end{array}$ & 1.66 \\
\hline hsa04310 & 'Wnt signaling pathway' & 0.3337 & 8 & $\begin{array}{l}\text { WNT7B, WNT7A, WIF1, FOSL1, } \\
\text { SFRP1, CAMK2B, etc. }\end{array}$ & 1.31 \\
\hline
\end{tabular}

MAPK, mitogen-activated protein kinase.

involved in different cellular processes, such as proliferation, survival, apoptosis, differentiation, motility and development (28). ERK, JNK/stress-activated protein kinase, and p38 are three major MAPK families that serve important roles in tumor progression in breast cancer $(29,30)$. KEGG pathway analysis showed that the differentially expressed genes, which were regulated by $H E P I S$, were functionally enriched in the MAPK and JAK-STAT signaling pathways. Therefore, HEPIS may regulate the progression of breast cancer by influencing the MAPK and JAK-STAT signaling pathways.

The IL-2/IL-2 receptor pathway is involved in the regulation of carcinoma cell proliferation, and endogenous IL-2 promotes growth and protects tumor cells from apoptosis (31). IFN/IFNAR2 leads to the phosphorylation of JAK/STAT and gene induction, as well as antiviral and antiproliferative cellular responses (32). In the present study, qPCR results revealed that IL2RA and IFNAR 2 were downregulated by HEPIS. Therefore, HEPIS may inhibit MCF-7 cell proliferation by regulating IL2RA and IFNAR2.

In conclusion, the results showed that HEPIS is primarily expressed in the cytoplasm of MCF-7 cells and inhibits the activity of the $N F-\kappa B$ reporter gene. Furthermore, the novel function of HEPIS was revealed as a proliferation inhibitor in breast cancer progression. Results from the gene chip analysis revealed that 2,231 genes were differentially expressed in the HEPIS overexpression group; these genes were enriched in the MAPK and JAK-STAT signaling pathways. The differential expression of the IL2RA, IFNAR2, and IFNA8 genes was confirmed using qPCR. Together, HEPIS may represent a novel target molecule for repression of breast cancer progression.

\section{Acknowledgements}

Not applicable.

\section{Funding}

This study was supported by grants from the General Higher Education Young Talents Program of Hebei Province (grant no. BJ2014027) and the Natural Science Foundation of Hebei Province (grant nos. H2018209140 and C2017209062). 


\section{Availability of data and materials}

The datasets used and/or analyzed during the present study are available from the corresponding author on reasonable request.

\section{Authors' contributions}

$\mathrm{FH}, \mathrm{YZ}$, and ML performed the in vitro experiments. $\mathrm{YZ}$ and YB analyzed the gene chip data. XZ performed the RNA in situ hybridization. $\mathrm{FH}$ and $\mathrm{XZ}$ were major contributors in writing the manuscript. All authors read and approved the final manuscript.

\section{Ethics approval and consent to participate}

Not applicable.

\section{Patient consent for publication}

Not applicable.

\section{Competing interests}

The authors declare that they have no competing interests.

\section{References}

1. Sawicki SG, Sawicki DL, Younker D, Meyer Y, Thiel V, Stokes H and Siddell SG: Functional and genetic analysis of coronavirus replicase-transcriptase proteins. PLoS Pathog 1: e39, 2005.

2. Hong M, Li W, Wang L, Jiang L, Liu L, Zhao H and Li Q: Identification of a novel transcriptional repressor (HEPIS) that interacts with nsp-10 of SARS coronavirus. Viral Immunol 21: 153-162, 2008.

3. Huttlin EL, Ting L, Bruckner RJ, Gebreab F, Gygi MP, Szpyt J, Tam S, Zarraga G, Colby G, Baltier K, et al: The BioPlex Network: A systematic exploration of the human interactome. Cell 162: 425-440, 2015.

4. Hu F and Zhang Y: Expression profile and promoter analysis of HEPIS. Exp Ther Med 15: 569-575, 2018.

5. Al-Hajj M, Wicha MS, Benito-Hernandez A, Morrison SJ and Clarke MF: Prospective identification of tumorigenic breast cancer cells. Proc Natl Acad Sci USA 100: 3983-3988, 2003.

6. Proceedings of the National Academy of Sciences of the United States of America: Annual subject and author indexes. Proc Natl Acad Sci USA 87 (Suppl): S10069-S10240, 1990.

7. Bray F, Ferlay J, Soerjomataram I, Siegel RL, Torre LA and Jemal A: Global cancer statistics 2018: GLOBOCAN estimates of incidence and mortality worldwide for 36 cancers in 185 countries. CA Cancer J Clin 68: 394-424, 2018.

8. Pike MC, Spicer DV, Dahmoush L and Press MF: Estrogens, progestogens, normal breast cell proliferation, and breast cancer risk. Epidemiol Rev 15: 17-35, 1993.

9. Braun S and Harbeck N: Molecular markers of metastasis in breast cancer: Current understanding and prospects for novel diagnosis and prevention. Expert Rev Mol Med 3: 1-14, 2001.

10. Darbre PD and King RJ: Steroid hormone regulation of cultured breast cancer cells. Cancer Treat Res 40: 307-341, 1988.

11. Hu F, Wang C, Du J, Sun W, Yan J, Mi D, Zhang J, Qiao Y, Zhu T and Yang S: DeltaEF1 promotes breast cancer cell proliferation through down-regulating p21 expression. Biochim Biophys Acta 1802: 301-312, 2010

12. Hu F, Meng X, Tong Q, Liang L, Xiang R, Zhu T and Yang S: BMP-6 inhibits cell proliferation by targeting microRNA-192 in breast cancer. Biochim Biophys Acta 1832: 2379-2390, 2013.
13. Hu F, Gou L, Liu Q, Zhang W, Luo M and Zhang X: G-patch domain containing 2 , a gene highly expressed in testes, inhibits nuclear factor-KB and cell proliferation. Mol Med Rep 11: 1252-1257, 2015

14. Liu Q, Song YJ, Meng LJ, Hu F, Gou LX, Jia CH, Tang HM, Wang WJ, Li M, Zhang XJ and Jia MC: Role of LM23 in cell proliferation and apoptosis and its expression during the testis development. Asian J Androl 15: 539-544, 2013.

15. Hu F, Yang S, Lv S, Peng Y, Meng L, Gou L and Zhang X: Analysis of AC3-33 gene expression in multiple organ cancer and adjacent normal tissue by RNA in situ hybridization. Oncol Lett 9: 2795-2798, 2015.

16. Zhao F, Pan S, Gu Y, Guo S, Dai Q, Yu Y and Zhang W: Small activating RNA restores the activity of the tumor suppressor HIC-1 on breast cancer. PLoS One 9: e86486, 2014.

17. R Core Team R: A language and environment for statistical computing. R Foundation for Statistical Computing, Vienna, Austria, 2012, https://www.R-project.org.

18. Zhang X, Hao L, Meng L, Liu M, Zhao L, Hu F, Ding C, Wang Y, He B, Pan Y, et al: Digital gene expression tag profiling analysis of the gene expression patterns regulating the early stage of mouse spermatogenesis. PLoS One 8: e58680, 2013.

19. Livak KJ and Schmittgen TD: Analysis of relative gene expression data using real-time quantitative PCR and the 2(-Delta Delta C(T)) method. Methods 25: 402-408, 2001.

20. Huxford T, Malek S and Ghosh G: Structure and mechanism in NF-kappa B/I kappa B signaling. Cold Spring Harb Symp Quant Biol 64: 533-540, 1999.

21. Baldwin AS Jr: The NF-kappa B and I kappa B proteins: New discoveries and insights. Annu Rev Immunol 14: 649-683, 1996.

22. Gebel HM, Braun DP, Tambur A, Frame D, Rana N and Dmowski WP: Spontaneous apoptosis of endometrial tissue is impaired in women with endometriosis. Fertil Steril 69: 1042-1047, 1998

23. Rawlings JS, Rosler KM and Harrison DA: The JAK/STAT signaling pathway. J Cell Sci 117: 1281-1283, 2004.

24. Igaz P, Toth S and Falus A: Biological and clinical significance of the JAK-STAT pathway; lessons from knockout mice. Inflamm Res 50: 435-441, 2001

25. O'Shea JJ, Gadina M and Schreiber RD: Cytokine signaling in 2002: New surprises in the Jak/Stat pathway. Cell 109 (Suppl): S121-S131, 2002

26. Kroon P, Berry PA, Stower MJ, Rodrigues G, Mann VM, Simms M, Bhasin D, Chettiar S, Li C, Li PK, et al: JAK-STAT blockade inhibits tumor initiation and clonogenic recovery of prostate cancer stem-like cells. Cancer Res 73: 5288-5298, 2013.

27. Hernandez-Vargas H, Ouzounova M, Le Calvez-Kelm F, Lambert MP, McKay-Chopin S, Tavtigian SV, Puisieux A, Matar C and Herceg Z: Methylome analysis reveals Jak-STAT pathway deregulation in putative breast cancer stem cells. Epigenetics 6: 428-439, 2011.

28. Plotnikov A, Zehorai E, Procaccia S and Seger R: The MAPK cascades: Signaling components, nuclear roles and mechanisms of nuclear translocation. Biochim Biophys Acta 1813: 1619-1633, 2011.

29. Davidson B, Konstantinovsky S, Kleinberg L, Nguyen MT, Bassarova A, Kvalheim G, Nesland JM and Reich R: The mitogen-activated protein kinases (MAPK) p38 and JNK are markers of tumor progression in breast carcinoma. Gynecol Oncol 102: 453-461, 2006.

30. Cocolakis E, Lemay S, Ali S and Lebrun JJ: The p38 MAPK pathway is required for cell growth inhibition of human breast cancer cells in response to activin. J Biol Chem 276: 18430-18436, 2001.

31. Reichert TE, Kashii Y, Stanson J, Zeevi A and Whiteside TL: The role of endogenous interleukin-2 in proliferation of human carcinoma cell lines. Br J Cancer 81: 822-831, 1999.

32. Schreiber G and Piehler J: The molecular basis for functional plasticity in type I interferon signaling. Trends Immunol 36: 139-149, 2015.

This work is licensed under a Creative Commons Attribution-NonCommercial-NoDerivatives 4.0 International (CC BY-NC-ND 4.0) License. 